\title{
Manual Removal of Primary Posterior Capsular Plaques Using Retinal End-Grasping Forceps
}

\author{
Gennady Landa (iD) Khoa Pham
}

Received: July 29, 2017 / Published online: September 21, 2017

(c) The Author(s) 2017. This article is an open access publication

\begin{abstract}
Introduction: Primary posterior capsular plaques can be highly adherent to the posterior capsule leading to possible posterior capsular rupture during attempted removal. Here we describe a novel method for posterior capsular plaque removal using a technique inspired by the experience of retina surgeon in the peeling of membranes from the posterior pole.

Methods: Retinal end-grasping forceps were used to peel the plaque from the posterior capsule.

Results: We have performed this technique on 12 eyes with successful removal of the posterior capsular plaque in all instances without compromise of the posterior capsule in any case.

Conclusions: We hope that the anterior segment surgeons will benefit by employing this
\end{abstract}

Enhanced content To view enhanced content for this article go to http://www.medengine.com/Redeem/ C90CF0601B3ADDD1.

G. Landa $(\bowtie) \cdot$ K. Pham

Department of Ophthalmology, New York Eye and Ear Infirmary of Mount Sinai, New York, NY, USA e-mail: doctor.landa@gmail.com; glanda@nyee.edu

G. Landa

Icahn School of Medicine at Mount Sinai, New York, NY, USA useful technique during cataract surgeries in the eyes with adherent posterior plaques.

Keywords: Cataract surgery; Posterior plaque; Retinal forceps

\section{INTRODUCTION}

Primary posterior capsular plaques pose a special challenge to cataract surgeons. The overall incidence of posterior capsular plaques can be as high as $10 \%$ [1]. The plaques can be highly adherent to the posterior capsule leading to possible posterior capsular rupture during attempted removal. As such, many surgeons prefer to leave posterior capsular plaques intact during cataract extraction and instead perform a YAG capsulotomy post-operatively to clear the visual axis. Alternatively, a number of techniques have been described to safely remove these plaques, including low vacuum automated irrigation and aspiration with or without the aid of special I/A tips and manual dissection of the plaque of the posterior capsule using viscoelastic. However, some of those plaques can be so adherent that those polishing techniques are often unable to be removed to clean the visual axis or even the violation of the posterior capsule may occur. Here, we describe a novel method for posterior capsular plaque removal using a technique inspired by the 
experience of retina surgeon in the peeling of membranes from the posterior pole.

\section{METHODS}

We evaluated retrospective case series of the technique, used for peeling of the posterior plaque that was an integrated part of a standard, uneventful cataract extraction surgery and did not change the plan of care. The study consisted of patients scheduled for elective cataract extraction procedure. All patients signed a general surgical consent. All procedures followed were in accordance with the ethical standards of the responsible committee on human experimentation (institutional and national) and with the Helsinki Declaration of 1964, as revised in 2013.

Following removal of all nuclear and cortical material, irrigation through the I/A tip can be continued to maintain the anterior chamber or, alternatively, a cohesive viscoelastic can be used to inflate the capsular bag kept deep in the anterior chamber. If the posterior plaque was present, retinal end-grasping forceps (Grieshaber Revolution ${ }^{\circledR}$ DSP Asymmetrical Forceps, $23 \mathrm{G}$ or $25 \mathrm{G}$ ) were then introduced through the paracentesis and used to peel the plaque from the posterior capsule. Since both 23G and 25G can be introduced into the anterior chamber through the $1 \mathrm{~mm}$ paracentesis, there is no need to enlarge the incision.

\section{RESULTS}

We have performed this technique on 12 eyes with successful removal of the posterior capsular plaque in all instances without compromise of the posterior capsule in any case. See enhanced content for video examples.

\section{DISCUSSION}

Primary posterior capsular plaques can be found in a variety of patients [1]. They are thought to be more common in elderly patients with mature cataracts, diabetic patients with advanced posterior subcapsular cataracts and pediatric patients [1]. Unlike in pediatrics patients where primary posterior capsulotomy is a desired outcome, adult patients with primary posterior subcapsular plaques present a dilemma for surgeons. While it is perfectly acceptable to leave posterior capsular plaques to undergo YAG capsulotomy post-operatively, a few negatives do accompany this approach. In addition to being another procedure for the patient, some posterior capsular plaques are not amenable to YAG capsulotomy, given their thickness and size.

As such, cataract surgeons are in need of a variety of techniques in their armamentarium to deal with posterior subcapsular plaques. Here, we describe a method that makes use of microforceps to peel away the posterior capsular plaque akin to what a retina surgeon would do during the peeling of epiretinal membranes.

A few advantages exist with this technique. During the technique both tactile and visual feedback are given to the surgeon to avoid rupture of the posterior capsule. In the event of a posterior capsule rupture, the rupture is likely to be confined to a small area, given the absence of flow or vacuum to extend the tear, as would be the case with I/A based techniques. Furthermore, the anterior hyaloid face would be more likely to be intact, preventing prolapse of the vitreous into the anterior chamber and obviating the need for an anterior vitrectomy. This technique is pivotal in combined cases when cataract surgery is performed along with retinal surgery, necessitating the use of intravitreal gas or silicon oil. In those cases, posterior capsule integrity and preservation of the separate compartments between anterior and posterior chambers is especially desirable. We assume that this technique would have its best results if performed by experienced cataract surgeons.

We advise using higher magnification while performing the peeling. Also, in order to facilitate the peeling, additional paracentesis may be created to allow a surgeon to use the dominant hand. As with all techniques, a learning curve exists for this technique, however, this technique is quite simple and can be easily performed by the anterior segment surgeons. 
There are some limitations, such as having a small number of cases and difficulty of standardization of surgical technique.

\section{CONCLUSIONS}

By describing this technique, we wanted to share the experience of the posterior segment surgeon with the anterior segment surgeons. We hope that this handy technique will be incorporated in the arsenal of the anterior segment surgeons in cases when it might be found relevant and useful.

\section{ACKNOWLEDGEMENTS}

No funding or sponsorship was received for this study or publication of this article. The article processing charges were funded by the authors. All named authors meet the International Committee of Medical Journal Editors (ICMJE) criteria for authorship for this manuscript, take responsibility for the integrity of the work as a whole, and have given final approval for the version to be published.

Disclosures. Gennady Landa, MD and Khoa Pham, MD have nothing to disclose.
Compliance with Ethics Guidelines. All procedures followed were in accordance with the ethical standards of the responsible committee on human experimentation (institutional and national) and with the Helsinki Declaration of 1964, as revised in 2013. All patients signed a general surgical consent.

Open Access. This article is distributed under the terms of the Creative Commons Attribution-NonCommercial 4.0 International License (http://creativecommons.org/licenses/ by-nc/4.0/), which permits any noncommercial use, distribution, and reproduction in any medium, provided you give appropriate credit to the original author(s) and the source, provide a link to the Creative Commons license, and indicate if changes were made.

\section{REFERENCE}

1. Vasovagal AR, Chauhan H, Shah G. Incidence of posterior capsular plaque in cataract surgery. J Cataract Refract Surg. 1997;23(5):798-802. 